\title{
Clinical Factors Associated with the Early Reduction of Corneal Sensitivity in Herpes Zoster Ophthalmicus
}

\author{
Minjeong Kim, Yeoun Sook Chun, Nam Ju Moon, Kyoung Woo Kim \\ Department of Ophthalmology, Chung-Ang University College of Medicine, Seoul, Korea
}

\begin{abstract}
Purpose: To identify clinical factors associated with the early reduction of corneal sensitivity (CS) in patients with herpes zoster ophthalmicus (HZO).

Methods: Total 30 subjects were divided into two groups according to the occurrence of an early reduction of CS (group 1, normal CS; group 2, decreased CS). The demographics, ocular manifestations of $\mathrm{HZO}$, and the ocular surface parameters were compared between two groups. Then, the relative risks of variables were analyzed to expect the early decrease of CS by $\mathrm{HZO}$ using logistic regression analysis.

Results: Among total 30 subjects, the CS decreased in 10 subjects ( $3.2 \pm 1.4 \mathrm{~cm}$, group 2$)$ and the other 20 subjects revealed normal CS (6.0 $\pm 0.0 \mathrm{~cm}$, group 1). The age was higher in the group 2 (67.0 \pm 16.6 years) than in group 1 (48.0 \pm 17.6 years, $p=0.013)$, but sex and the comorbidity of diabetes mellitus was not difference between groups. The presence of corneal punctate epithelial erosions was a single factor among the ocular manifestations of HZO, which discriminated group 1 (5\%) and group 2 (60\%, $p=0.002)$. The ocular surface staining scores (i.e., National Eye Institute scores and Sjögren's International Collaborative Clinical Alliance ocular surface score) were higher in group 2 ( $p=0.001$ and $p=0.021$, respectively) than in group 1. The existence of corneal punctate epithelial erosions revealed as a sole risk factor for the early reduction of CS (odds ratio $=33.085 ; p=0.016)$.

Conclusions: It is necessary to pay attention to the possible occurrence of neurotrophic keratopathy especially in older patients with $\mathrm{HZO}$ showing an initial presence of corneal punctate epithelial erosions.
\end{abstract}

Key Words: Corneal punctate epithelial erosions, Corneal sensitivity, Herpes zoster ophthalmicus

Herpes zoster ophthalmicus (HZO) is a disease of reactivation of latent varicella zoster virus (VZV) throughout the ophthalmic branch of the fifth cranial nerve (CN V) [1].

Received: October 15, 2021 Final revision: December 27, 2021 Accepted: January 18, 2022

Corresponding Author: Kyoung Woo Kim, MD, PhD. Department of Ophthalmology, Chung-Ang University College of Medicine, 84 Heukseok-ro, Dongjak-gu, Seoul 06973, Korea. Tel: 82-2-6299-1689, Fax: 82-2825-1666, E-mail: kkanssa@cau.ac.kr
HZO causes typical symptoms including sharp neuralgia and vesicular eruption along the area of a single dermatome of $\mathrm{CN}$ V1. The eyeball involvements range from $20 \%$ to $90 \%$ in patients with HZO and they present as keratitis, conjunctivitis, anterior uveitis, scleritis, and optic neuritis [2-6]. Amongst, keratitis and conjunctivitis have been reported as a common manifestation of HZO in previous studies [2-8].

As the sensory nerve innervation of the cornea travels in the nasociliary nerve and its long ciliary nerve branches, 
recurrent inflammatory reactions caused by viral infection along the nerve can destroy the corneal sensitivity (CS). It was known that a single episode of HZO can cause a profound loss of CS [9]. Despite VZV is neurotropic and so has a predilection for residing latently in the peripheral nervous system, the clinically acknowledged neurotrophic keratopathy may develop as low as in $25 \%$ of HZO patients [10]. However, regarding that the persistent reduction of CS is an important factor in the occurrence of chronic epithelial lesions or neurotrophic keratopathy with visual loss $[8,11]$, the early notice for the corneal nerve involvement of VZV and the reduction of CS may be clinically beneficial.

Punctate epithelial keratitis and pseudodendrites are commonly seen as early and transient findings with the presence of VZV antigen on the ocular surface [1,12]. In addition, nummular anterior stromal infiltration or keratouveitis can develop representing direct viral infection or an immune reaction [1]. These findings are easily noticed by slit-lamp examinations in clinics. By contrast, we should rely on the use of an esthesiometer or confocal microscopy to judge the corneal nerve involvement of HZO before a typical oval-shaped corneal ulcer for neurotrophic keratitis occurs or a persistent epithelial defect is accompanied by. When it is hard to assess the corneal nerve damage due to the absence of the examination tools, clinicians may be faced with an obvious and advanced type of neurotrophic keratitis unexpectedly. If clinicians may predict the future occurrence of HZO-related neurotrophic keratopathy with clinical clues, it would be very useful to establish the follow-up interval accordingly and to notify patients the possible aggravation of keratitis.

In this regard, herein, we compared the demographics, manifestations for the eye involvement of $\mathrm{HZO}$, and ocular surface parameters between HZO patients with and without the early reduction of CS to identify clinical factors which are associated with the early reduction of CS in patients with HZO.

\section{Materials and Methods}

\section{Ethics statement}

This study was a retrospective comparative study of patients diagnosed with HZO. The whole process properly followed the tenets of the Declaration of Helsinki. The study was approved by the institutional review board of Chung-Ang University Hospital (No. 2008-061-19325). Written informed consent was waived by the institutional review board due to the retrospective nature of the study.

\section{Subjects}

A total of 30 subjects who satisfy diagnostic criteria of HZO from May 2019 until May 2021 at Chung-Ang University Hospital were included $\mathrm{HZO}$ was defined as the presence of typical vesicular rashes affecting the $\mathrm{CN}$ V1 dermatome. Facial zoster was first diagnosed by dermatologists in all subjects, thereafter they were referred to an ophthalmology clinic to be evaluated for HZO within 1 week. The presence of eye involvement of HZO and the ocular manifestations were evaluated by a single ophthalmologist (KWK). Subjects were divided into two groups according to their corneal sensitivity: HZO with normal CS (i.e., group 1) and $\mathrm{HZO}$ with an ipsilateral reduction of CS (i.e., group 2). Demographics and clinical presentations were reviewed and analyzed.

\section{Study design}

In this retrospective study, we searched for the clinical factors which were related with the early reduction of CS in HZO subjects. For this, we used various independent variables including age, the comorbidity of type 2 diabetes mellitus (DM), the manifestations for the eye involvement of HZO and ocular surface parameters, to investigate their contribution to the decreased CS which was a dependent variable.

\section{Measurement of CS}

The CS was measured within 1 month after the diagnosis of HZO and was measured at the corneal center using a Cochet-Bonnet esthesiometer (Luneau Technology, Chartres, France). Starting from the longest length, $6 \mathrm{~cm}$, the test was performed by decreasing the length of the monofilament that touches the center of the cornea by $0.5 \mathrm{~cm}$. When a subject felt a touch, the length of the monofilament was recorded as the threshold $(\mathrm{cm})$ of the CS. If a subject did not acknowledge a touch, the length of monofilament was reduction in increments of $0.5 \mathrm{~cm}$ until the subject gave a positive response. 


\section{Ocular surface parameters}

Ocular surface parameters including matrix metalloproteinase (MMP)-9, tear secretion, corneal and conjunctival surface staining, and meibomian gland dysfunction (MGD) profiles was evaluated at the diagnosis of HZO in ophthalmology clinics.

The test for tear MMP-9 was done with a point-of-care immunoassay kit (InflammaDry; Quidel, San Diego, CA,

Table 1. Demographics of groups with and without early reduction of corneal sensitivity in subjects of herpes zoster ophthalmicus

\begin{tabular}{lccc}
\hline Variable & $\begin{array}{c}\text { Group 1 } \\
(\mathrm{n}=20)\end{array}$ & $\begin{array}{c}\text { Group 2 } \\
(\mathrm{n}=10)\end{array}$ & $p$-value \\
\hline $\begin{array}{l}\text { Corneal sensitivity } \\
(\mathrm{cm})\end{array}$ & $6.0 \pm 0.0$ & $3.2 \pm 1.4$ & $<0.001^{\ddagger}$ \\
$\begin{array}{l}\text { Age }(\mathrm{yr}) \\
\text { Sex }\end{array}$ & $48.0 \pm 17.6$ & $67.0 \pm 16.6$ & $0.013^{\ddagger}$ \\
$\quad$ Male & $13(65)$ & $5(50)$ & 0.461 \\
Female & $7(35)$ & $5(50)$ & \\
Diabetes mellitus & & & 0.076 \\
Yes & $2(10)$ & $4(40)$ & \\
No & $18(90)$ & $6(60)$ & \\
\hline
\end{tabular}

Values are presented as mean \pm standard deviation or number (\%).

"Normal corneal sensitivity; ${ }^{\circ}$ Decreased corneal sensitivity;

${ }^{\ddagger}$ Statistically significant.
USA) which was previously demonstrated to properly diagnose inflammation related to dry eye, following instructions from product documentation. Tear MMP-9 expression was grade into 0 to 4 based on a 5-stage grading system [13]. Tear secretion was determined by Schirmer I test. Schirmer standard strip (Eagle Vision, Memphis, TN, USA) was applied on the outer $1 / 3$ point of lower conjunctival fornix and absorbed tear fluid for 5 minutes without analgesia. For ocular staining score, each eye went through slit-lamp examination under yellow filter after fluorescein instillation. Corneal and conjunctival erosions were evaluated according to the National Eye Institute scoring system [14] and Sjögren's International Collaborative Clinical Alliance (SICCA) ocular surface score (OSS) only at the conjunctival area [15], respectively.

Evaluation for MGD was done through dynamic observation of meibomian glands at the upper lids: meibum expressibility throughout meibomian gland (MG) orifices (i.e., MG expressibility grade) and meibum quality (i.e., meibum quality grade). MG expressibility grade was evaluated with firm digital pressure at upper central lid margin and was graded from 0 to 3: 0 , all glands expressible; 1, 3-4 glands expressible; 2, 1-2 glands expressible; and 3, no glands expressible $[16,17]$. Meibum quality was graded from 0 to 3 after squeezing upper central meibomian glands: 0 , clear secretion; 1 , cloudy fluid; 2 , cloudy particulate fluid; and 3, toothpaste-like expression [17].

Table 2. Ocular involvement and the ocular manifestations of HZO in groups with and without early reduction of corneal sensitivity

\begin{tabular}{|c|c|c|c|c|c|c|c|c|}
\hline \multirow[b]{2}{*}{$\begin{array}{l}\text { Ocular } \\
\text { involvement }\end{array}$} & \multicolumn{6}{|c|}{ Ocular manifestations of HZO } & \multicolumn{2}{|c|}{ No. of subjects $(\%)$} \\
\hline & $\begin{array}{l}\text { Skin lesions } \\
\text { at CN V1 } \\
\text { dermatome }\end{array}$ & $\begin{array}{c}\text { Corneal } \\
\text { pseudodendrites }\end{array}$ & $\begin{array}{c}\text { Corneal punctate } \\
\text { epithelial } \\
\text { erosions }\end{array}$ & $\begin{array}{c}\text { Corneal } \\
\text { stromal } \\
\text { infiltration }\end{array}$ & $\begin{array}{l}\text { Anterior } \\
\text { uveitis }\end{array}$ & Episcleritis & $\begin{array}{l}\text { Group 1* } \\
(n=20)\end{array}$ & $\begin{array}{r}\text { Group } 2^{\dagger} \\
(\mathrm{n}=10)\end{array}$ \\
\hline \multirow[t]{9}{*}{$+(\mathrm{n}=17)$} & + & + & + & + & + & - & $1(5)$ & $0(0)$ \\
\hline & + & + & + & - & + & - & $0(0)$ & $1(10)$ \\
\hline & + & + & + & - & - & - & $0(0)$ & $1(10)$ \\
\hline & + & + & - & + & + & - & $3(15)$ & $0(0)$ \\
\hline & + & + & - & - & + & - & $0(0)$ & $2(20)$ \\
\hline & + & + & - & - & - & - & $3(15)$ & $1(10)$ \\
\hline & + & - & + & - & + & - & $0(0)$ & $1(10)$ \\
\hline & + & - & + & - & - & - & $0(0)$ & $3(30)$ \\
\hline & + & - & - & - & - & + & $1(5)$ & $0(0)$ \\
\hline$-(n=13)$ & + & - & - & - & - & - & $12(60)$ & $1(10)$ \\
\hline
\end{tabular}

$\mathrm{HZO}=$ herpes zoster ophthalmicus; $\mathrm{CN} \mathrm{V}=$ the fifth cranial nerve; + = present; - = absent.

${ }^{*}$ Normal corneal sensitivity; ${ }^{\dagger}$ Decreased corneal sensitivity. 


\section{Statistical analysis}

For statistical analysis, Prism software ver. 8.4.3 (GraphPad, La Jolla, CA, USA) was used. To compare data between two independent groups, we used a Mann-Whitney $U$-test and a chi-square test. To analyze the contributory effect of independent variable on the early reduction of $\mathrm{CS}$, the logistic regression test was used. The data are ex-

Table 3. Difference of ocular manifestations of herpes zoster ophthalmicus between groups with and without early reduction of corneal sensitivity

\begin{tabular}{lccc}
\hline Variable & $\begin{array}{c}\text { Group 1* } \\
(\mathrm{n}=20)\end{array}$ & $\begin{array}{c}\text { Group 2 } \\
(\mathrm{n}=10)\end{array}$ & $p$-value \\
\hline $\begin{array}{l}\text { Corneal } \\
\text { pseudodendrites }\end{array}$ & & & 0.461 \\
$\quad$ Yes & $7(35)$ & $5(50)$ & \\
$\quad$ No & $13(65)$ & $5(50)$ & \\
$\begin{array}{l}\text { Corneal punctate } \\
\text { epithelial erosions }\end{array}$ & & & $0.002^{\ddagger}$ \\
$\quad$ Yes & $1(5)$ & $6(60)$ & \\
No & $19(95)$ & $4(40)$ & \\
$\begin{array}{l}\text { Corneal stromal } \\
\text { infiltration }\end{array}$ & & & 0.272 \\
$\quad$ Yes & $4(20)$ & $0(0)$ & \\
$\quad$ No & $16(80)$ & $10(100)$ & \\
Anterior uveitis & & & 0.384 \\
$\quad$ Yes & $4(20)$ & $4(40)$ & \\
No & $16(80)$ & $6(60)$ & \\
\hline
\end{tabular}

Values are presented as number (\%).

"Normal corneal sensitivity; 'Decreased corneal sensitivity; ${ }^{\ddagger}$ Statistically significant. pressed as mean \pm standard deviation, and the differences were considered statistically significant at $p<0.05$.

\section{Results}

\section{Demographics}

Demographics in each group are described in Table 1. The average CS was $6.0 \pm 0.0 \mathrm{~cm}$ in group 1 and was $3.2 \pm$ $1.4 \mathrm{~cm}$ in group $2(p<0.001)$. The average age was significantly higher in group $2(67.0 \pm 16.6$ years) than in group 1 (48.0 \pm 17.6 years, $p=0.013$ ). The sex and the comorbidity of type $2 \mathrm{DM}$ was not different between groups ( $p=0.461$ and $p=0.076$, respectively).

\section{Difference of manifestations for the eye involvement of $\mathrm{HZO}$ between groups}

The presence of the ocular involvement and its manifestations are described in detail in Table 2. Ocular involvement was observed in 17 subjects (56.7\%) among total subjects and the other 13 subjects (43.3\%) revealed only skin lesions at CN V1 dermatome area with absence of ocular manifestations (Table 2). The average CS in subjects with the ocular involvement $(4.4 \pm 1.7 \mathrm{~cm})$ was significantly lower than that in subjects without the ocular involvement $(5.7 \pm 1.1 \mathrm{~cm}, p=0.019)$. Interestingly, one subject in group 2 whose CS decreased to $2.0 \mathrm{~cm}$ did not reveal the ocular involvement of $\mathrm{HZO}$.

The existence of each ocular manifestation for HZO was compared between groups (Table 3). The population with

Table 4. Difference of the ocular surface parameters between groups with and without early reduction of corneal sensitivity in subjects of herpes zoster ophthalmicus

\begin{tabular}{lccc}
\hline Variable & Group $1^{*}$ & Group 2 & $p$-value \\
\hline Tear MMP-9 (Gr) & $1.8 \pm 1.3$ & $2.8 \pm 1.1$ & 0.138 \\
Schirmer I without anesthesia (mm) & $15.1 \pm 10.5$ & $22.2 \pm 9.4$ & 0.112 \\
Corneal punctate erosions (score) & $0.5 \pm 0.9$ & $3.2 \pm 1.9$ & $0.001^{\ddagger}$ \\
SICCA OSS & $0.3 \pm 0.6$ & $1.2 \pm 0.8$ & $0.021^{\ddagger}$ \\
MG expressibility (Gr) & $1.4 \pm 0.8$ & $1.7 \pm 0.5$ & 0.283 \\
Meibum quality (Gr) & $1.1 \pm 0.7$ & $1.7 \pm 0.5$ & 0.146 \\
\hline
\end{tabular}

Values are presented as mean \pm standard deviation.

MMP = matrix metalloproteinase; SICCA = Sjögren's International Collaborative Clinical Alliance; OSS = ocular surface score; MG = meibomian gland.

${ }^{*}$ Normal corneal sensitivity; ${ }^{\dagger}$ Decreased corneal sensitivity; ${ }^{\dagger}$ Statistically significant. 
corneal punctate epithelial erosions $(60 \%)$ was significantly higher in group 2 than that $(5 \%)$ in group $1(p=0.002)$. The presence of corneal pseudodendrites, corneal stromal infiltration, or anterior uveitis did not differ between groups ( $p=0.461, p=0.272$, and $p=0.384$, respectively).

\section{Difference of the ocular surface parameters between groups}

The difference of the ocular surface parameters including tear MMP-9 expression, tear secretion by Schirmer I, ocular surface erosions, and clinical grades for MGD was compared between groups (Table 4). The corneal punctate erosion score and SICCA OSS were significantly higher in group 2 than in group 1 ( $p=0.001$ and $p=0.021$, respectively). The tear MMP-9 grade, tear secretion, MG expressibility grade and meibum quality grade were not difference between groups ( $p=0.138, p=0.112, p=0.283$, and $p=0.146$, respectively).

\section{Relative contributor for the early reduction of CS in $\mathrm{HZO}$}

Next, we evaluated the contributing effects of age and the existence of corneal punctate epithelial erosions which revealed inter-group difference and the comorbidity of DM which had marginal significance of difference between groups on the early reduction of CS in HZO (Table 5). According to the logistic regression analysis, the age and the comorbidity of DM were not related with the early occurrence of the decrease of CS ( $p=0.116$ and $p=0.085$ ) but the existence of corneal punctate epithelial erosions was significantly correlated with the early reduction of CS (odds ratio, 33.085; $p=0.016$ ).

\section{Discussion}

In this study, we identified that the presence of corneal punctate epithelial erosions and higher age was associated with an occurrence of the early reduction of CS in patients with HZO. Other well-known ocular manifestations of HZO including corneal epithelial pseudodendrites, corneal stromal infiltration, and anterior uveitis were not related to the decrease of CS. Although it is known that the corneal punctate epithelial erosions are one of the early features of neurotrophic keratitis, we stress that the stromal keratitis and pseudodentritic epithelial inflammation would not be closely related with the clinically noticeable corneal nerve damage unexpectedly.

Damage along the nerve fiber caused by the repopulation of VZV leads to the breakdown of stability of corneal epithelium that results in abnormally elevated epithelium which gathers together to form pseudodendritic lesions [18]. Interestingly, although pseudodendritic lesions of the cornea are one of the characteristic features of HZO, it was not significantly related to the development of impaired corneal sense in our study. The reason is still enigmatic. But we speculate that diffuse punctate epithelial erosions may reflect the existence of a higher load of VZV which might spread out through multiple corneal nerve branches and arrive in wide areas on the corneal surface. By contrast, sparse pseudodendritic lesions or sectorial stromal infiltrative foci might be the results of the localized involvement of corneal nerve branches by viruses unlike in the corneal punctate epithelial erosion. This issue can be confirmed by identification of the area of the damaged corneal nerve using confocal microscopy in a future study.

Demographically, the proportion of the older aged was significantly higher in the group of the reduction of CS in this study (Table 1). The aging process impairs the sensory nerve regeneration after its damage [19]. It is unclear that

Table 5. Logistic regression analysis describing the effect of variables on the early reduction of corneal sensitivity in subjects of herpes zoster ophthalmicus

\begin{tabular}{lccccc}
\hline Dependent variable & $\beta$ & SE & Wald & \multicolumn{1}{c}{$p$-value } & Odds ratio \\
\hline Age & 0.058 & 0.037 & 2.476 & 0.116 & 1.060 \\
Diabetes mellitus & 2.298 & 1.334 & 2.967 & 0.085 & 9.951 \\
Corneal punctate epithelial erosions & 3.499 & 1.458 & 5.757 & $0.016^{*}$ & 33.085 \\
\hline
\end{tabular}

$\mathrm{SE}=$ standard error.

*Statistically significant.. 
the corneal peripheral nerves from the subjects with higher age may be more easily affected with VZV or maybe less regenerated after the VZV repopulation. However, we propose that the initial presentation of corneal punctate epithelial erosions should not be overlooked after the diagnosis of HZO, especially in an elderly patient.

The viral infection including herpes zoster is the most common cause of corneal insensitivity that can eventually result in neurotrophic keratopathy [20]. Impaired corneal sensory innervation leads to instability of cornea as proper distribution of nerve is important in maintaining the suitable environment of the cornea including metabolism and mitosis of epithelial cells. While several studies have shown that the changes in the levels of neuro-mediators such as substance $\mathrm{P}$ and acetylcholine in the animal cornea after the nerve injury might cause the refractory epithelial defect [20,21], a neurotropic keratopathy often lacks signs and symptoms [20]. According to Mackie classification, the stage I neurotrophic ulcer is just characterized by epithelial irregularity most commonly in the form of punctate keratopathy without epithelial defect [22]. Because the punctate keratopathy is easily seen in diverse ocular surface diseases such as dry eye disease, toxic keratopathy, and MGD, clinicians may be biased on the observation of pseudodendritic lesions and keratouveitis in HZO patients. Despite the early manifestations of HZO-related neurotrophic keratopathy resembles with those of dry eyes, they may lead to persistent corneal epithelial defects, severe inflammation, corneal melting, and potentially perforation as it aggravates [23]. In contrast to herpes simplex virus keratitis, however, profound loss of corneal sensation can occur in a single episode of HZO, and these ulcers are more likely to perforate. Therefore, it is important to remind clinicians to be concerned about corneal punctate epithelial erosions as a possible clinical marker for the reduction of CS despite other ocular manifestations for the eye involvement of HZO such as corneal epithelial pseudodendrites which is more specific to $\mathrm{HZO}$ are absent.

This study is limited by its retrospective nature and the small sample size. In addition, it was impossible to evaluate the longitudinal change of the CS in subjects with early reduction of CS. Because ocular manifestations could develop weeks to months after the initial diagnosis of HZO [6], our cross-sectional analysis of dry eye-related ocular parameters and the presence of ocular manifestations at the diagnosis of HZO might not reflect the pathogenic role of corneal nerve damage in HZO. Unfortunately, in this study with a small sample size, we could not investigate the relevance of the existence of multiple ocular manifestations with the reduction of CS compared to that of the existence of a particular single manifestation. Nevertheless, this is the first study that focused on identifying clinical and ocular factors associated with the early reduction of CS which is a main pathophysiologic role in HZO. Advanced understanding through a future study will aid clinicians in guiding further care of HZO patients with ocular involvement.

In conclusion, the initial presence of corneal punctate epithelial erosions and the older age may be associated with the early reduction of CS in HZO. It is necessary to pay attention to the possible occurrence of neurotrophic keratopathy in those patients.

\section{Conflicts of Interest: None.}

Acknowledgements: None.

Funding: None.

\section{References}

1. Liesegang TJ. Herpes zoster ophthalmicus natural history, risk factors, clinical presentation, and morbidity. Ophthalmology 2008;115(2 Suppl):S3-12.

2. Borkar DS, Tham VM, Esterberg E, et al. Incidence of herpes zoster ophthalmicus: results from the Pacific Ocular Inflammation Study. Ophthalmology 2013;120:451-6.

3. Edell AR, Cohen EJ. Herpes simplex and herpes zoster eye disease: presentation and management at a city hospital for the underserved in the United States. Eye Contact Lens 2013;39:311-4.

4. Kahloun R, Attia S, Jelliti B, et al. Ocular involvement and visual outcome of herpes zoster ophthalmicus: review of 45 patients from Tunisia, North Africa. J Ophthalmic Inflamm Infect 2014:4:25.

5. Szeto SK, Chan TC, Wong RL, et al. Prevalence of ocular manifestations and visual outcomes in patients with herpes zoster ophthalmicus. Cornea 2017;36:338-42.

6. Yawn BP, Wollan PC, St Sauver JL, Butterfield LC. Herpes zoster eye complications: rates and trends. Mayo Clin Proc 2013;88:562-70.

7. Ghaznawi N, Virdi A, Dayan A, et al. Herpes zoster ophthalmicus: comparison of disease in patients 60 years and 
older versus younger than 60 years. Ophthalmology 2011;118:2242-50.

8. Nithyanandam S, Stephen J, Joseph M, Dabir S. Factors affecting visual outcome in herpes zoster ophthalmicus: a prospective study. Clin Exp Ophthalmol 2010;38:845-50.

9. Yang AY, Chow J, Liu J. Corneal innervation and sensation: the eye and beyond. Yale J Biol Med 2018;91:13-21.

10. Liesegang TJ. Corneal complications from herpes zoster ophthalmicus. Ophthalmology 1985;92:316-24.

11. Liesegang TJ. Varicella-zoster virus eye disease. Cornea 1999;18:511-31.

12. Pavan-Langston D, Yamamoto S, Dunkel EC. Delayed herpes zoster pseudodendrites: polymerase chain reaction detection of viral DNA and a role for antiviral therapy. Arch Ophthalmol 1995;113:1381-5.

13. Kim M, Oh JY, Bae SH, et al. Assessment of reliability and validity of the 5-scale grading system of the point-of-care immunoassay for tear matrix metalloproteinase-9. Sci Rep 2021;11:12394.

14. Lemp MA. Report of the National Eye Institute/Industry workshop on clinical trials in dry eyes. CLAO J 1995;21: 221-32.

15. Whitcher JP, Shiboski CH, Shiboski SC, et al. A simplified quantitative method for assessing keratoconjunctivitis sicca from the Sjogren's Syndrome International Registry. Am J Ophthalmol 2010;149:405-15.
16. Pflugfelder SC, Tseng SC, Sanabria O, et al. Evaluation of subjective assessments and objective diagnostic tests for diagnosing tear-film disorders known to cause ocular irritation. Cornea 1998;17:38-56.

17. Tomlinson A, Bron AJ, Korb DR, et al. The international workshop on meibomian gland dysfunction: report of the diagnosis subcommittee. Invest Ophthalmol Vis Sci 2011;52:2006-49.

18. Lee HJ, Kim SY, Jung MS. The clinical characteristics of facial herpes zoster in Korean patients. $J$ Korean Ophthalmol Soc 2010;51:8-13.

19. Yu FS, Yin J, Lee P, et al. Sensory nerve regeneration after epithelium wounding in normal and diabetic cornea. Expert Rev Ophthalmol 2015;10:383-92.

20. Bonini S, Rama P, Olzi D, Lambiase A. Neurotrophic keratitis. Eye (Lond) 2003;17:989-95.

21. Versura P, Giannaccare G, Pellegrini M, et al. Neurotrophic keratitis: current challenges and future prospects. Eye Brain 2018;10:37-45.

22. Mackie IA. Neuroparalytic keratitis. In: Fraunfelder F, Roy FH, Meyer SM, editors. Current ocular therapy. Philadelphia: WB Saunders; 1995. p. 452-4.

23. Hamrah P, Cruzat A, Dastjerdi MH, et al. Unilateral herpes zoster ophthalmicus results in bilateral corneal nerve alteration: an in vivo confocal microscopy study. Ophthalmology 2013;120:40-7. 